\title{
Bayesian Image Segmentation Using Local Iso-Intensity Structural Orientation
}

\author{
Wilbur C. K. Wong and Albert C. S. Chung
}

\begin{abstract}
Image segmentation is a fundamental problem in early computer vision. In segmentation of flat shaded, nontextured objects in real-world images, objects are usually assumed to be piecewise homogeneous. This assumption, however, is not always valid with images such as medical images. As a result, any techniques based on this assumption may produce less-than-satisfactory image segmentation. In this work, we relax the piecewise homogeneous assumption. By assuming that the intensity nonuniformity is smooth in the imaged objects, a novel algorithm that exploits the coherence in the intensity profile to segment objects is proposed. The algorithm uses a novel smoothness prior to improve the quality of image segmentation. The formulation of the prior is based on the coherence of the local structural orientation in the image. The segmentation process is performed in a Bayesian framework. Local structural orientation estimation is obtained with an orientation tensor. Comparisons between the conventional Hessian matrix and the orientation tensor have been conducted. The experimental results on the synthetic images and the real-world images have indicated that our novel segmentation algorithm produces better segmentations than both the global thresholding with the maximum likelihood estimation and the algorithm with the multilevel logistic MRF model.
\end{abstract}

Index Terms-Biomedical image processing, Hessian matrices, image segmentation, Markov processes, maximum a posteriori (MAP) estimation, maximum likelihood estimation, spatial data structures, stochastic fields.

\section{INTRODUCTION}

$\mathbf{I}$ MAGE segmentation is one of the fundamental problems in early computer vision. It is a process to partition an image into nonoverlapping regions. To extract high-level information from an image, digital image analysis systems, such as industrial inspection systems, autonomous object recognition systems, and medical image analysis systems, may need to accomplish image segmentation prior to any post-processing algorithms [1]. As such, a tremendous amount of thorough research has taken place on image segmentation [2]-[4].

Approaches to image segmentation can be categorized into two different perspectives: 1) edge based and 2) region based. Edge-based approaches segment an image by taking the edge information into account. Edge pixel is denoted by intensity

\footnotetext{
Manuscript received January 6, 2004; revised September 14, 2004. This work was supported in part by the Hong Kong Research Grants Council (HK RGC) under Grants HKUST6209/02E and DAG01/02.EG04 and in part by the Sino Software Research Institute (SSRI) under Grant SSRI01/02.EG22. The associate editor coordinating the review of this manuscript and approving it for publication was Dr. Zoltan Kato.

The authors are with the Lo Kwee-Seong Medical Image Laboratory and the Department of Computer Science, The Hong Kong University of Science and Technology, Kowloon, Hong Kong (e-mail: cswilbur@cs.ust.hk; achung@cs.ust.hk).

Digital Object Identifier 10.1109/TIP.2005.852199
}

discontinuity in the image. It can be detected by a differential operator such as Sobel, Roberts, Prewitt, and Laplacian operators [2], [4]. An edge linking algorithm has been employed to assemble broken edge pixels into interconnected boundaries, and the image is partitioned into disjointed regions (i.e., segmented) according to these boundaries [1]. Apart from the explicit usage of edge pixel, gradient information has been used to drive evolving contours with the level set methods and topologically adaptable surfaces to segment objects in images [3], [5]-[8]. With these techniques, the evolving contours and surfaces are attracted to locations with a high gradient, i.e., the presence of an edge. Moreover, a watershed algorithm has proven to be a useful tool for image segmentation with edge information [9].

In region-based approaches, an image is divided into different groups of pixels (i.e., classes or regions) according to a certain similarity criterion. For example, techniques based on histogramming and multilevel thresholding assume that image intensity values are independent and identically distributed (i.i.d.) and that the pixels in the same region have similar intensity values. These techniques usually segment an image based on some estimated intensity distributions. Statistical mixture modeling [10], k-means algorithm [11], and fuzzy clustering algorithm [12]-[14] are examples of these techniques. There are other region-based techniques, namely, region growing, split and merge algorithm, relaxation labeling, and Markov random field (MRF)-based and neural network-based approaches, which partition an image with reference to both an intensity similarity criterion and spatial information [1], [15]-[21].

In this paper, we are interested in the binary segmentation of a flat shaded, nontextured three-dimensional (3-D) object. This type of object in the real-world image is usually assumed to be piecewise constant with random white Gaussian noise contamination [2], [4], [22]. In other words, in the noiseless image (i.e., the truth image), pixels that belong to the same object should have the same intensity value. The boundaries between objects are well-defined by sharp changes in the intensity profile. The authors of the aforementioned segmentation techniques demonstrated that if the piecewise constant assumption is valid and the signal-to-noise ratio (SNR) is reasonably high, their methods should be capable of giving satisfactory image segmentation.

However, the assumption of piecewise constant is not always valid in the real-world image. Examples of the violation of the assumption are commonly found in medical images such as intensity inhomogeneity in magnetic resonance imaging ${ }^{1}$ (MRI)

\footnotetext{
${ }^{1}$ Intensity inhomogeneity in MRI may relate to poor radio frequency coil uniformity or operating condition of the MR scanners [23].
} 
and low-intensity vascular region in phase-contrast magnetic resonance angiography ${ }^{2}$ (PC MRA). Pixels that belong to the same tissue class in an MRI may have different intensity values owing to the intensity inhomogeneity. Due to the low-intensity profile near the vascular wall in a PC MRA, any edges detected by a differential operator or the Laplacian operator in the image may not delineate the true vascular boundary.

Despite the intensity nonuniformity within the imaged object, changes in the intensity values are usually smooth. In other words, the image data surface (i.e., the intensity profile) is coherent (see [25] for more information). In the literature, several authors have exploited this property to segment images with explicit modeling of the smooth image data surface. Besl and Jain [25] demonstrated the application of the variable-order surface fitting algorithm to segment objects with a set of curved surfaces modeled by bivariate polynomials. Leclerc [26] employed a smooth function that is defined in terms of the Taylor coefficients in the spatial domain to describe the data surface coherence. The author claimed that this description is the simplest and the most stable description that can be applied to the image partitioning problem. Tu and Zhu [27] formulated the observation model in their Bayesian segmentation algorithm with a two-dimensional (2-D) Bezier-spline model. This model aims at representing an inhomogeneous pattern in an image that corresponds to a shading effect over space. An explicit model in the spatial domain is used to represent the data surface coherence among all the approaches. As pointed out in [25] and [27], these explicit modeling techniques can be applied to the images with sufficiently large regions (more than 10-30 pixels).

In this paper, a novel algorithm that exploits the image data surface coherence is proposed. This algorithm is capable of representing the coherence in a relatively small local region. We follow a research line [28] in which spatial information is incorporated into the image segmentation process as a prior probability within an MRF framework. Our formulation of the data surface coherence is different from the one proposed by $\mathrm{Tu}$ and Zhu [27]. Instead of embedding the coherent information in the observation model as proposed in [27], we embed it in an MRF prior model. Furthermore, the image data surface coherence is estimated in the Fourier domain, as opposed to the techniques mentioned in the previous paragraph, which used an explicit model and an error function to approximate the surface coherence.

Because the image data surface is coherent, iso-intensity structures may be found in the image. These structures characterize the local property of the image data surface. They are locally coherent as long as the image data surface is coherent (see Section II-C for the illustration). The newly proposed method exploits this local structural coherence to improve the quality of the binary segmentation of an image. A new smoothness prior model, namely, local structural orientation smoothness prior, is proposed to provide regularization on the image segmentation. The formulation of the prior is based on

\footnotetext{
${ }^{2}$ Intensity in the speed image of PC MRA is proportional to the speed of the blood flow in the imaged vessels. Because of blood viscosity, the blood flow is low near the vascular boundary and inside an aneurysm (a vascular disease due to local abnormal dilatation of blood vessel) [24]. As a result, image intensity values are low in these vascular regions.
}

the orientation smoothness of the iso-intensity structures in the image. To demonstrate the applicability of the novel smoothness prior model, we have tested the proposed algorithm on both synthetic and real-world images. The binary segmentation is approximated with a finite mixture model (FMM) and the iterated conditional modes (ICM) in the Bayesian framework.

The rest of the paper is organized as follows. In Section II, the Bayesian approach to the binary segmentation of objects with smooth intensity inhomogeneity is presented. A robust method to estimate the local structural orientation of the coherent image data surface is described in Section III. We also present a comparison of another conventional method for the estimation of the local structural orientation in the same section. The proposed image segmentation algorithm is outlined in Section IV. Sensitivity analyses of the MRF parameters are presented in Section V. The experimental results on the synthetic images and real-world images are given in Section VI, followed by a discussion of the algorithm performance. Finally, conclusions are drawn in Section VII.

\section{BAYESIAN APPROACH to SEgMENTING ObJECTS With COHERENT INTENSITY PROFILE}

In this section, we formulate the binary segmentation problem in the Bayesian framework. The observation model and the newly proposed prior model are presented. The prior model, namely, local structural orientation smoothness prior, acts as a contextual constraint on the segmentation of the objects with a coherent intensity profile.

\section{A. Problem Formulation}

Assume that a problem of object binary segmentation can be considered as a process of assigning labels from a label set $\mathcal{L}=\{$ background, object $\}$ to each of the voxels indexed in $\mathcal{S}=\{1, \ldots, m\}$, where $m$ is the total number of voxels in an observed image $\vec{y}$. Let a vector $\vec{x}$ be a binary segmentation of the image $\vec{y}$, then each element in the vector $\vec{x}$ can be regarded as a mapping from $\mathcal{S}$ to $\mathcal{L}$, i.e., $x_{i}: \mathcal{S} \rightarrow \mathcal{L}$. A feasible segmentation $\vec{x}$ is, therefore, in a Cartesian product $\Omega_{x}$ of $m$ label sets. The set $\Omega_{x}$ is known as the configuration space. In the Bayesian framework, the optimal solution is given by a feasible segmentation $\vec{x}^{*}$ of the image $\vec{y}$, which maximizes the posterior probability $p(\vec{x} \mid \vec{y}) \propto p(\vec{y} \mid \vec{x}) p(\vec{x})$ over the space $\Omega_{x}$ [22]. The likelihood probability $p(\vec{y} \mid \vec{x})$ can be application specific. It suggests particular label assignments based on the intensity values in the image $\vec{y}$. While the prior probability $p(\vec{x})$ constrains the binary segmentation contextually. The likelihood and the prior probabilities are also known as the observation and the prior models in the Bayesian segmentation framework.

In order to have a tractable constraint, the MRF theory is used. By virtue of the Hammersley-Clifford theorem [29], the Gibbs distribution provides us with a practical way of specifying the joint probability (i.e., the prior probability $p(\vec{x})$ ) of an MRF. Therefore, the maximum a posteriori (MAP) estimate $\vec{x}^{*}$ becomes a minimum of the summation of the likelihood energy and the prior energy functions over the configuration space $\Omega_{x}$

$$
\vec{x}^{*}=\arg \min _{\vec{x} \in \Omega_{x}}(U(\vec{y} \mid \vec{x})+U(\vec{x}))
$$


where $U(\vec{y} \mid \vec{x})=-\log p(\vec{y} \mid \vec{x})$ represents the likelihood energy function and $U(\vec{x})=\sum_{c \in \mathcal{C}} V_{c}(\vec{x})$ is the prior energy function, which is a sum of clique potentials $V_{c}(\vec{x})$ over all possible cliques in $\mathcal{C} \subseteq \mathcal{S}$ [22].

\section{B. Observation Model}

In practice, because of the high complexity of the random variables $\vec{x}$ and $\vec{y}$, it is computationally intractable to calculate the likelihood energy $U(\vec{y} \mid \vec{x})$ from the negative log-likelihood $(-\log p(\vec{y} \mid \vec{x}))$. Therefore, it is usually assumed that the intensity values in the image $\vec{y}$ are independent and identically distributed (i.i.d.), and can be modeled by a FMM [10]. The calculation of the likelihood energy then becomes tractable and can be estimated with the FMM, since the global likelihood is determined by local likelihoods. The likelihood energy function can be rewritten as

$$
U(\vec{y} \mid \vec{x})=-\sum_{i \in S} \log p\left(y_{i} \mid x_{i}\right)
$$

\section{Prior Model}

This paper focuses on the binary segmentation of the 3-D objects with a coherent intensity profile (i.e., smooth changes in the intensity values) and does not consider any textured objects. Imagine the object image is inspected on a microscopic level, within a tiny spatial window for example, we can observe isosurfaces in the 3-D image. These iso-surfaces depict the structures with the same intensity values. For legibility, the above idea is illustrated with a 2-D ring object in Fig. 1. Fig. 1(a) shows the 2-D ring with a coherent intensity profile. Fig. 1(b) plots the image data surface (i.e., intensity profile) of the ring. Fig. 1(c) shows the iso-contours on the image data surface. Fig. 1(d) shows the closeup of the square region in Fig. 1(a). The corresponding closeups of the image data surface and the iso-contours are shown in Fig. 1(e) and (f). It is observed that each iso-contour is locally coherent and is as smooth as the corresponding image data surface. This implies that the local orientation of each iso-contour line fragment is also coherent. Similar observation can be made in the 3-D space. The local orientation of each iso-surface planar patch is also coherent.

In this section, a new smoothness prior model that exploits the local structural coherence of the image data surface is presented. The prior model is used to constrain the binary segmentation within the Bayesian framework. Smoothness constraints have been used to solve low-level vision problems, including surface reconstruction, optical flow determination and shape extraction. These applications demonstrate that the generic contextual constraint is a useful prior to a variety of low-level vision problems [22]. In the MRF framework, a contextual constraint is expressed as a prior probability or the equivalent prior energy function $U(\vec{x})$ as given in (1).

In the formulation of the prior energy function, the afore-discussed local structural coherence is exploited. The prior energy function is expressed as

$$
U(\vec{x})=\sum_{i \in \mathcal{S} j \in \mathcal{N}_{i}}\left(1-f\left(x_{i}\right)\right) f\left(x_{j}\right) g(i, j)\left(\beta_{1} h_{1}(i, j)+\beta_{2} h_{2}(i, j)\right)
$$

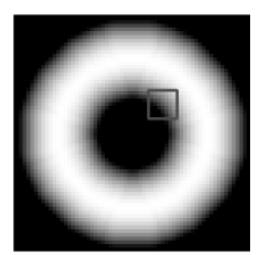

(a) Image

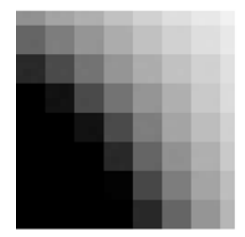

(d) Close-up

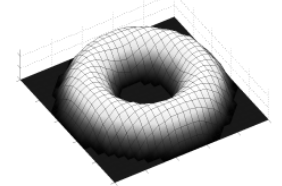

(b) Data surface

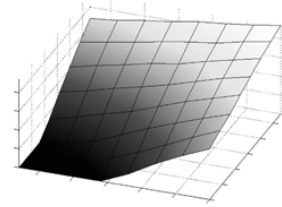

(e) Data surface

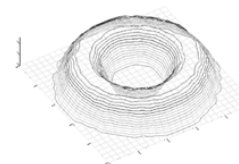

(c) Iso-contour

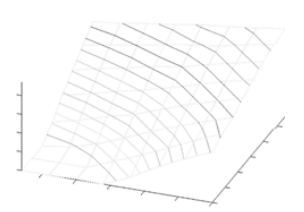

(f) Iso-contour
Fig. 1. Two-dimensional ring image. (a) The ring with a coherent intensity profile. (b) Image data surface of the ring. (c) Iso-contours on the image data surface. (d) Closeup of the square region in (a). (e) Image data surface of the closeup region. (f) Iso-contours of the closeup region.

where $\mathcal{N}_{i}$ denotes a set of voxels adjacent to voxel $i$ with respect to a neighborhood system $\mathcal{N} \subseteq \mathcal{S}$ and $f$ is a mapping function defined as

$$
f\left(x_{i}\right)= \begin{cases}0, & x_{i}=\text { background } \\ 1, & x_{i}=\text { object }\end{cases}
$$

$g(i, j)$ measures the geometric closeness (Euclidean distance) between voxels $i$ and $j$, which defines the structural locality; $h_{1}(i, j)$ and $h_{2}(i, j)$ measure the orientation similarities (quantitation of the structural coherence) of the first and the second principal directions of the iso-surface planar patch at voxel $i$ with respect to voxel $j$, respectively; $\beta_{1}$ and $\beta_{2}$ are positive weights, which need not sum to one and are used to control the influence of orientation coherence in the interactions between the adjacent voxels. The idea of applying geometric closeness and similarity measures as constraints is similar to the one found in the bilateral filters [30].

In this paper, the geometric closeness $g$ and the orientation similarity measures $h_{1}$ and $h_{2}$ are Gaussian functions of the magnitude of the relative position vector of voxel $j$ from voxel $i,\left\|\vec{u}_{i j}\right\|$, and the orientation discrepancy $\delta$, between voxels $i$ and $j$, respectively. The geometric closeness function is given as a decreasing function $g$ when the distance $\left\|\vec{u}_{i j}\right\|$ increases

$$
g(i, j)=\exp \left(\frac{-\left\|\vec{u}_{i j}\right\|^{2}}{2 \sigma_{g}^{2}}\right)
$$

where $\sigma_{g}$ defines the desired structural locality between neighboring voxels. The function $g \rightarrow 1$ if voxel $j$ is very close to voxel $i$; otherwise, $g$ decreases to zero as the Euclidean distance between voxels $i$ and $j$ increases. The orientation similarity function $h_{k}$ is written as a decreasing function when the orientation discrepancy $\delta$ increases

$$
h_{k}(i, j)=\exp \left(\frac{-\delta^{2}\left(\hat{\overrightarrow{\vec{u}}}_{i j}, \hat{\vec{w}}_{k}\right)}{2 \sigma_{h}^{2}}\right)
$$

where $k=1,2$, the functions $h_{1}$ and $h_{2}$ denote the orientation similarities of the first and the second principal directions of 
the iso-surface planar patch at voxel $i$ with respect to voxel $j$, respectively. The notation $\hat{\vec{u}}$ denotes the unit vector of vector $\vec{u}$. The orientation discrepancy function $\delta$ is defined as

$$
\delta(\vec{u}, \vec{v})=1-\left|\vec{u}^{T} \vec{v}\right| .
$$

The vectors $\hat{\vec{w}}_{1}$ and $\hat{\vec{w}}_{2}$ depict the first and the second principal directions of iso-surface planar patch at voxel $i$, respectively, and the parameter $\sigma_{h}$ is chosen based on the desired amount of orientation discrepancy filtering among adjacent voxels. It is noted that the function $\delta \rightarrow 0$ if vectors $\vec{u}$ and $\vec{v}$ are aligned, i.e., the angle between $\vec{u}$ and $\vec{v}$ equals either $0^{\circ}$ or $180^{\circ}$. As such, the function $h_{k} \rightarrow 1$ if voxel $j$ is located along one of the principal directions of the planar patch at voxel $i$, as depicted by the vectors $\hat{\vec{w}}_{1}$ and $\hat{\vec{w}}_{2}$.

To summarize, the prior energy function in (3) encourages piecewise continuous object label assignment in the segmentation. Piecewise continuity is constrained by the geometric closeness and the structural orientation similarity measures. Fig. 2 demonstrates two scenarios in the label assignment process. On one hand, if the voxels $i$ and $j$ are close enough, i.e., $g \rightarrow 1$, the label assigned to the voxel $j$ is object, i.e., $f\left(x_{j}\right)=1$, and the voxel $j$ is aligned with the first or the second principal direction of the iso-surface planar patch at the voxel $i$ [as illustrated in Fig. 2(a)], i.e., $h_{k} \rightarrow 1, k \in\{1,2\}$, it is in favor of object label assignment to the voxel $i$, i.e., setting $f\left(x_{i}\right)=1$. This is because we are minimizing the energy function in (3). On the other hand, if the label assigned to the voxel $j$ is background, i.e., $f\left(x_{j}\right)=0$, as indicated in Fig. 2(b), the prior energy vanishes and the label assignment to the voxel $i$ is based solely on the likelihood energy.

In other words, the prior model described in this section assumes the object is smooth with respect to its iso-surfaces. The structural coherence is not extended to the background class because it is assumed that the background is structureless and is contaminated by random white noise.

\section{Estimating Local Structural ORIENTATION By Eigen DECOMPOSITION OF ORIENTATION TENSOR}

As discussed in Section II, the two principal directions of the iso-surface planar patches (i.e., $\hat{\vec{w}}_{1}$ and $\hat{\vec{w}}_{2}$ ) are exploited to constrain the binary segmentation of a 3-D object with an MRF prior model. In this section, we describe a robust method to estimate the two principal directions. The estimation is obtained from an orientation tensor rather than a conventional Hessian matrix for better performance and the robustness to noise. Experimental results of the performance comparisons between the two methods are presented.

\section{A. Orientation Tensor}

The use of an orientation tensor for local structure description was first presented in Knutsson's work [31]. The work was motivated by the need to find a continuous representation of local orientation. Knutsson formulated the orientation tensor by combining outputs from a number of directional polar separable quadrature filters.

A quadrature filter is constructed in the Fourier domain. It is a complex valued filter in the spatial domain. The real and

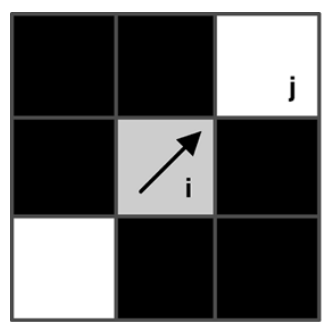

(a) Scenario 1

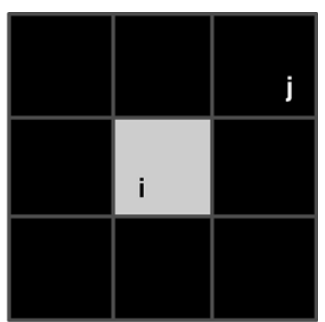

(b) Scenario 2
Fig. 2. Label assignment scenarios. (a) Scenario 1: The voxel $j$ is aligned with the first or the second principal direction (arrow) of the iso-surface planar patch at the voxel $i$ and the label assigned to the voxel $j$ is object (white box). (b) Scenario 2: The label assigned to the voxel $j$ is background (black box).

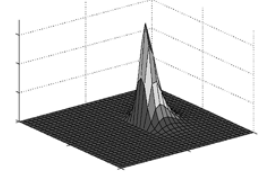

(a) Fourier domain

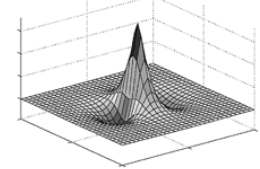

(b) Real part in spatial domain

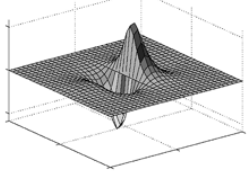

(c) Imaginary part in spatial domain
Fig. 3. Quadrature filter in a 2-D space. (a) The quadrature filter in the Fourier domain. (b) Real part of the quadrature filter in the spatial domain. It is observed that the filter responses are symmetric. It is known as a line filter in the spatial domain. (c) Imaginary part of the quadrature filter in the spatial domain. It is observed that the filter responses are antisymmetric. It is known as an edge filter in the spatial domain.

the imaginary parts of the complex value give the symmetric and the antisymmetric filter responses, respectively. Therefore, a quadrature filter can be viewed as a pair of filters in the spatial domain: 1) a line filter that corresponds to the symmetric response and 2) an edge filter that corresponds to the antisymmetric response. Furthermore, the quadrature filter is orientation specific. This implies that the filter is sensitive to lines and edges that are orientated in the filter direction. Fig. 3 shows a 2-D quadrature filter in the Fourier domain as well as its real and imaginary parts in the spatial domain.

In Knutsson's formulation, the orientation tensor $\mathbf{T}$ in a 3-D space is defined, based on six quadrature filters, as follows:

$$
\mathbf{T}=\sum_{k=1}^{6} q_{k}\left(\frac{5}{4} \hat{\vec{n}}_{k} \hat{\vec{n}}_{k}^{T}-\frac{1}{4} \mathbf{I}\right)
$$

where $q_{k}$ is the modulus of the complex valued response from the quadrature filter in the direction $\hat{\vec{n}}_{k}$ and $\mathbf{I}$ is the identity tensor. For further details and the definitions of the six quadrature filters (see [31] or [32, Ch. 6]).

\section{B. Local Orientation Estimation}

Estimation of local structural orientation is performed via eigen decomposition of the orientation tensor $\mathbf{T}$ at each voxel in an image [32]. To calculate the tensor $\mathbf{T}$, the image should be convolved with the six quadrature filters. After the convolutions, there are six moduli of the complex valued filter responses associated with each voxel, $q_{k}, k=1,2, \ldots, 6$. Then, the tensor is computed as stated in (8).

Let $\lambda_{1}, \lambda_{2}$ and $\lambda_{3}$ be the eigenvalues of the tensor $\mathbf{T}$ in descending order $\left(\lambda_{1} \geq \lambda_{2} \geq \lambda_{3} \geq 0\right)$ and $\hat{\vec{e}}_{i}(i=1,2,3)$ be 
the corresponding eigenvectors, respectively. The estimation of the local structural orientation can be based on one of the following three cases. (a) Planar case: $\lambda_{1} \gg \lambda_{2} \simeq \lambda_{3}, \hat{\vec{e}}_{2}$ and $\hat{\vec{e}}_{3}$ represent the estimates to the principal directions of the planar structure. (b) Linear case: $\lambda_{1} \simeq \lambda_{2} \gg \lambda_{3}, \hat{\vec{e}}_{3}$ is an estimate to the principal direction of the linear structure. (c) Isotropic case: $\lambda_{1} \simeq \lambda_{2} \simeq \lambda_{3}$, no specific orientation.

As outlined in Section II-C, we exploit the first and the second principal directions of the iso-surface planar patches in the MRF prior model [i.e., $\hat{\vec{w}}_{1}$ and $\hat{\vec{w}}_{2}$ in (6)]. With the eigen decomposition of the orientation tensors, we can then approximate the two principal directions with the eigenvectors $\hat{\vec{e}}_{3}$ and $\hat{\vec{e}}_{2}$, respectively. Fig. 4 summarizes the local orientation estimation process in a flow diagram.

\section{Comparison of Orientation Tensor With Hessian Matrix}

Using an orientation tensor is not the only approach to estimating local structural orientation. A Hessian matrix (defined as in [33]) can also be used for the estimation (see [34] and [35]). In this section, we compare the performance of the two aforementioned approaches.

A synthetic ring torus image with a parabolic ${ }^{3}$ intensity profile (peak intensity equals 255 at the center of the tube) in a volume of size $64 \times 64 \times 64$ voxels has been built. The radius from the center of the hole to the center of the torus tube is 20 voxels and the radius of the tube is 10 voxels. A complete 3-D surface model of the torus image is shown in Fig. 5(a) and a clipped torus model is shown in Fig. 5(b). The clipped model is cut vertically at the 21 st slice for a better illustration of the corresponding slice image and its segmentation. Fig. 5(c) and (d) shows the 21 st slice image of the torus and its Truth segmentation, respectively.

We have compared the performance of the orientation tensor and the Hessian matrix approaches (hereafter referred to as "OT" and "HESSIAN," respectively) on the truth (i.e., noiseless) torus and noisy toruses at different levels of additive white Gaussian noise. SNR is defined as the ratio of the peak intensity value to the sample standard deviation of the noise. Fig. 5(e) shows the 21 st slice image of the noisy torus with $\mathrm{SNR}=5$ (i.e., the sample standard deviation equals 51 in the Gaussian noise). Comparison is based on the orientation discrepancy [function $\delta$ in (7)] between the estimated and the truth iso-surface normals. ${ }^{4}$

In all the experiments on the performance comparison, the following configurations have been used. In OT, a $5 \times 5 \times 5$ filter window with relative bandwidth $B$ equals 2 and center frequency $\rho$ equals $\pi / 2 \sqrt{2}$ has been used (relative bandwidth $B$ and center frequency $\rho$ control the characteristics of the quadrature filters, see [32] for further details). This configuration of the quadrature filters is taken from [32], since the optimization of the filter parameters is not the focus of this work. A $3 \times 3$ Gaussian kernel with $\sigma=1$ has been employed for tensor averaging (for further details, see [32, Ch. 6]). The Gaussian kernel accords with the size of the orientation tensor in the 3-D space,

\footnotetext{
${ }^{3} \mathrm{~A}$ parabolic model is used because of its simplicity to model coherent intensity profile within a tubular object in a 3-D space.

${ }^{4}$ We may think of the tube-axis-symmetric intensity in the torus image as the sliding of a series of concentric tubes in different intensity values. The isosurface normals are referred to as the surface normals of these concentric tubes.
}

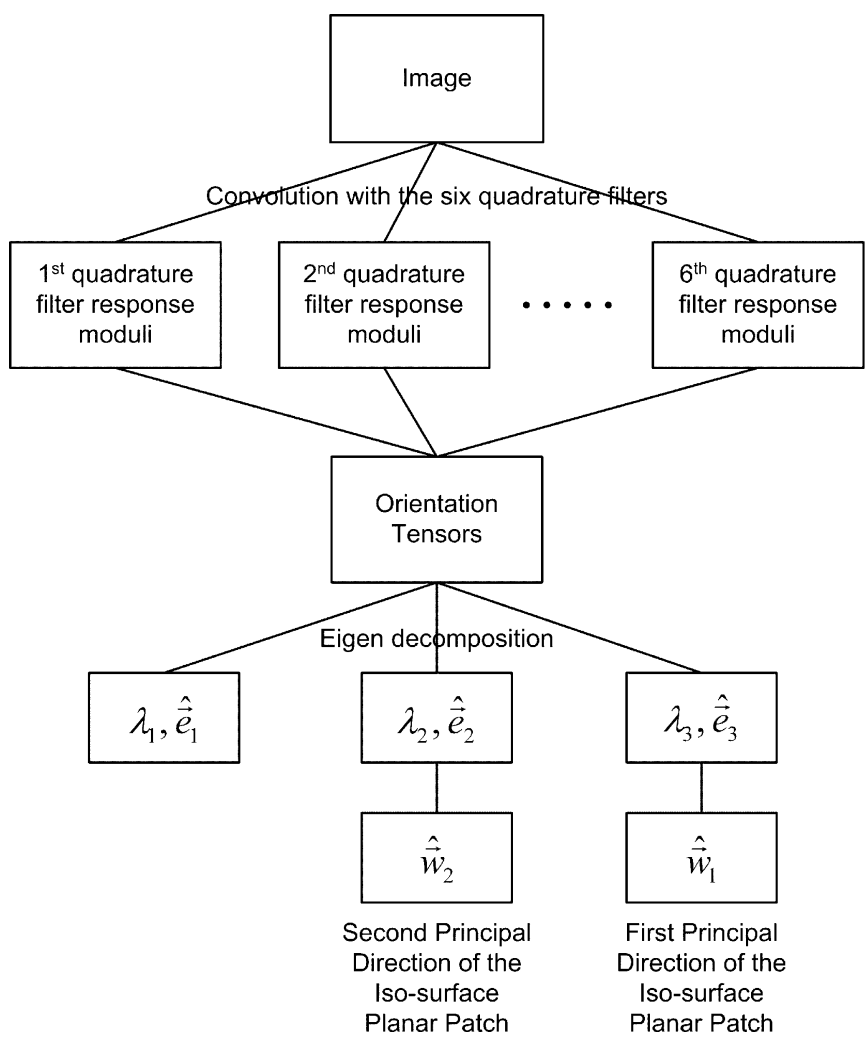

Fig. 4. Flow diagram of the local orientation estimation process.

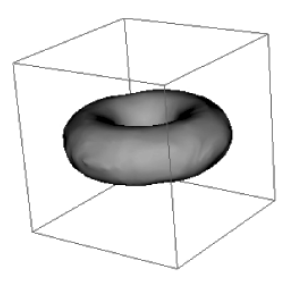

(a) Complete model

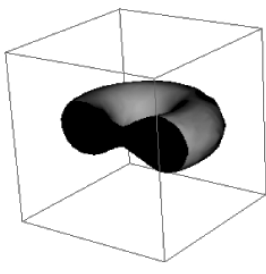

(b) Clipped model

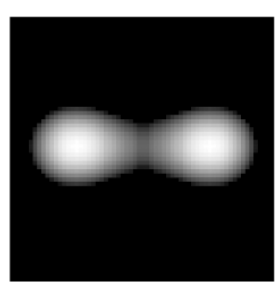

(c) Noiseless

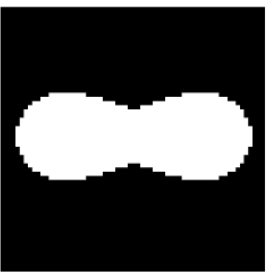

(d) Truth

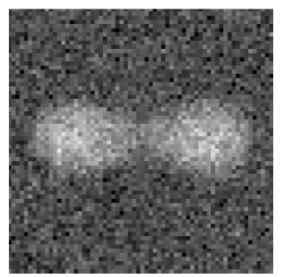

(e) Noisy
Fig. 5. Three-dimensional synthetic image, ring torus. (a) Complete 3-D surface model of the torus image, (b) clipped 3-D surface model cut vertically at the 21 st slice, (c) the 21 st slice image of the torus, its corresponding (d) truth segmentation, and (e) noisy image with $\mathrm{SNR}=5$.

which is a $3 \times 3$ matrix. In HESSIAN, a $5 \times 5 \times 5$ Gaussian kernel with $\sigma=5 / 3$ and the central finite difference approximation have been used. For the sake of adequate comparison, the size of this Gaussian kernel is chosen to match the quadrature filter window size employed in OT.

Fig. 6(a) shows the statistics of the orientation discrepancy estimated by the two approaches on the truth torus. It is observed that OT produces most of the estimations which fall into the minor discrepancy region (left-hand-side of the vertical dotted 


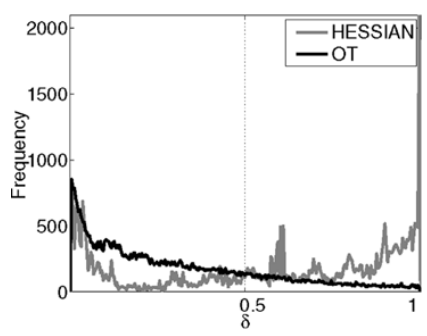

(a) Noiseless torus

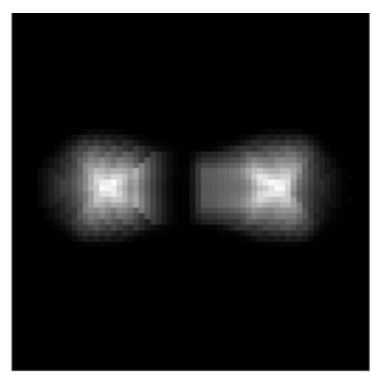

(b) OT

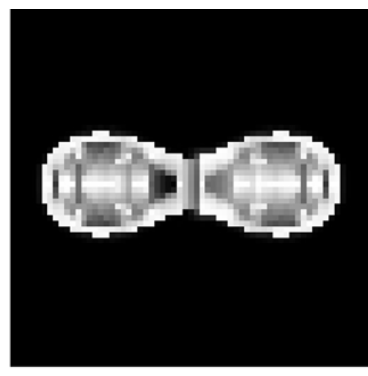

(c) HESSIAN

Fig. 6. (a) Statistics of the orientation discrepancy estimated by the orientation tensor approach ("OT") and the Hessian matrix approach ("HESSIAN") on the noiseless ring torus. (b) and (c) Orientation discrepancies shown on a slice image. Orientation discrepancy is computed based on the function $\delta$ in (7) between the estimated and the truth iso-intensity surface normals. The surface normals are obtained from (b) OT and (c) HESSIAN. White pixel denotes discrepancy equals 1 , i.e., the largest discrepancy.

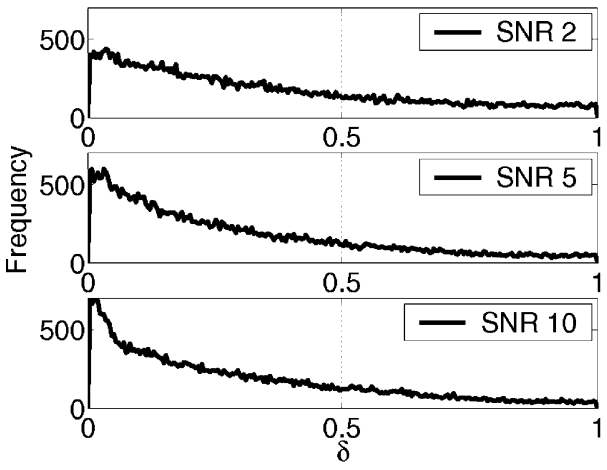

(a) OT

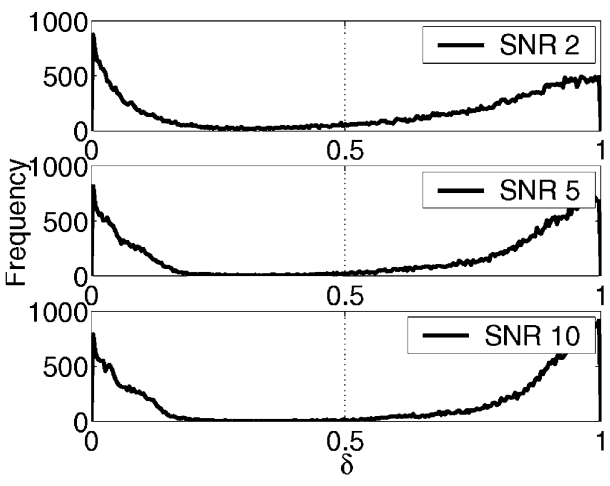

(b) HESSIAN

Fig. 7. Statistics for the discrepancy of the orientation estimated with (a) OT and (b) HESSIAN on the ring torus with noise at different levels, SNR $=2,5,10$.

line in the graph $\delta<0.5$, i.e., $<60^{\circ}$ angular difference with the truth iso-surface normal). HESSIAN, on the other hand, gives most of the estimations which fall onto the major discrepancy region (right-hand-side of the vertical dotted line in the graph).

Fig. 6(b) and (c) shows the orientation discrepancies obtained from OT and HESSIAN, respectively, on a slice image (bright voxel indicates the location of large orientation discrepancy). It is evident that HESSIAN gives large discrepancies throughout the ring torus. Conversely, OT only produces large discrepancies near the center of the torus tube, where the local structural orientations are hard to be determined because of the close-toconstant intensity profile at the tube center. ${ }^{5}$ These experimental results have shown that the orientation estimated by HESSIAN is less than satisfactory. It may be due to the application of the (first order neighborhood) central finite difference approximation in HESSIAN. On the contrary, OT demonstrates adequate orientation estimations by taking the advantage of estimation in the Fourier domain.

Fig. 7(a) and (b) shows the orientation discrepancies among ring toruses with different noise levels, $\mathrm{SNR}=2,5,10$, for OT and HESSIAN, respectively. It is noted that OT is more robust to noise than HESSIAN. OT is capable of giving most of the estimations within the minor discrepancy region even for the image corrupted by severe noise (i.e., SNR $=2$ ).

${ }^{5}$ If a 3-D image has a constant intensity profile, it is unable to determine the orientation of any iso-surface planar patches in the image. This is because the image itself is structureless.
All these experimental results demonstrate that the orientation tensor approach to the local structural orientation estimation exhibits better performance as compared with the conventional Hessian matrix approach.

\section{MAIN AlgORITHM}

The estimation of the model parameters and local structural orientation, and the approximation to the optimal solution are discussed in this section. The initialization of the approximation algorithm follows. As a summary, Algorithm 1 outlines the binary segmentation algorithm proposed in this paper.

Algorithm 1 Main algorithm

1: Estimate the local structural orientation with an orientation tensor, compute $\hat{\vec{e}}_{3}$ and $\hat{\vec{e}}_{2}$ (i.e., $\hat{\vec{w}}_{1}$ and $\hat{\vec{w}}_{2}$ ) and the likelihood probability $p\left(y_{i} \mid x_{i}\right)$ at each voxel. 2: Initialize the algorithm with the ML estimate $\vec{x}^{0}, k \Leftarrow 0$.

3: Repeat.

4: $\quad k \Leftarrow k+1$.

5: For all $i$ in the set $\mathcal{S}$, do

6: $\quad E_{o} \Leftarrow-\log p\left(y_{i} \mid\right.$ object $)$.

$7:$

$$
\begin{array}{r}
E_{b} \Leftarrow \sum_{j \in \mathcal{N}_{i}} f\left(x_{j}^{k-1}\right) g(i, j)\left(\beta_{1} h_{1}(i, j)+\beta_{2} h_{2}(i, j)\right) \\
-\log p\left(y_{i} \mid \text { background }\right) .
\end{array}
$$


8: If $E_{o}>E_{b}$, then

9: $\quad x_{i}^{k} \Leftarrow$ background.

10: $\mathbf{E l s e}$

11: $\quad x_{i}^{k} \Leftarrow$ object.

12: End if

13: End for

14: Until convergence.

15: Return the final segmentation $\vec{x}^{k}$.

\section{A. Parameters Estimation}

There are several free parameters in the segmentation algorithm presented in Section II. In the observation model [i.e., the likelihood energy function $U(\vec{y} \mid \vec{x})$ in (2)], the free parameters are dependent on the choice of FMM, which is application specific. In this paper, the FMM employed in the experiments presented in Sections VI-A and VI-B are the Gaussian-uniform (GU) and the Maxwell-Gaussian-uniform (MGU) mixture models, respectively (see the corresponding section for further details). The FMM free parameters can be estimated by the expectation-maximization (EM) algorithm as discussed in [10].

On the other hand, in the MRF prior model [i.e., the prior energy function $U(\vec{x})$ in (3)], there are four free parameters, viz. $\sigma_{g}, \sigma_{h}, \beta_{1}$, and $\beta_{2}$, namely, the MRF parameters. In general, there are two approaches to estimating the MRF parameters [22]: 1) supervised estimation with labeled data and 2) unsupervised estimation with unlabeled data.

The term "supervised" refers to the fact that the solution of the problem is known and is used to estimate the unknown parameters. In the context of this paper, this means the binary segmentations (i.e., the labeled data) of the images are employed in the estimation. From the viewpoint of machine learning, such supervised estimation is a learning with training data. Pseudolikelihood approximation scheme, the coding method, mean field approximation, least squares fit procedure and Markov chain Monte Carlo (MCMC) methods can be used in this kind of supervised estimation (see [22, Ch. 6.1] for detailed discussions). In the case of unsupervised estimation, several authors have adopted an iterative labeling-estimation strategy [36]-[40]. In their approaches, segmentation and free parameter estimation are performed alternately. In this work, the MRF parameters are found empirically. The sensitivity of the MRF parameters in image binary segmentations is analyzed and will be presented in Section V.

\section{B. Estimating Local Structural Orientation}

To estimate the local structural orientation with an orientation tensor, as outlined in Section III, six quadrature filters of a window size $5 \times 5 \times 5$, relative bandwidth $B$ equal to 2 and center frequency $\rho$ equal to $\pi / 2 \sqrt{2}$ are used. A 3-D image is convolved with the filters to obtain six moduli of complex valued responses at each voxel. Then the orientation tensor is computed as given in (8). The eigen decomposition of the orientation tensor is performed and the two eigenvectors $\hat{\vec{e}}_{2}$ and $\hat{\vec{e}}_{3}$, which approximate the principal directions of the iso-surface planar patch, are obtained at each voxel.

\section{Approximation to the Optimal Solution}

As shown in [41], the energy minimization problem given in (1) is NP hard. It is inefficient to compute the global minimum. Therefore, we opt for a fast approximation to the optimal solution with local minimum. In the literature, a variety of optimization algorithms have been demonstrated to approximate the solution in energy minimization problems. These algorithms include simulated annealing (SA) algorithm ${ }^{6}$ [42], ICM [29], graduated nonconvexity (GNC) [43], mean field annealing (MFA) algorithm [28], [44], [45], maximizer of the posterior marginals (MPM) estimator [46], graph cuts algorithm [41], [47], and inference algorithm on quadtree [38] (see [22, Ch. 8 and 9] for further discussions).

However, not all the aforementioned optimization algorithms are capable of optimizing arbitrary energy functionals. For example, in the graph cuts algorithm [41], [47], the energy functionals should be metric or semimetric (see [41] for the definition of metric and semimetric), whereas, in the inference algorithm on quadtree [38], the functionals should be formulated on a Markov chain.

Furthermore, although most of the authors have demonstrated that their optimization algorithms are applicable to a wide range of functionals [28], [43]-[46], there has been very limited attention given to the optimization of nonsymmetrical inhomogeneous energy functionals. The functional proposed in this paper [see (3)] is one of these kinds. To the best of our knowledge, SA and ICM are two of the optimization algorithms that are competent to optimize the newly proposed functional.

ICM is chosen to solve the minimization problem because of its fast convergence to the solution approximation. Moreover, our observation and prior models are entirely based on local information (see Sections II-B and II-C for details), and our initial estimate of the binary segmentation can be very close to the optimal solution. These make ICM more preferable to other optimization algorithms, particularly in time-critical applications (for instance, medical image analysis applications).

\section{Algorithm Initialization}

To initialize the ICM algorithm, a natural choice is the maximum likelihood (ML) estimate, as suggested in [22]. Given the fact that the parameters of the observation model are known, the initial binary segmentation $\vec{x}^{0}$ is obtained as follows:

$$
\vec{x}^{0}=\left\{\arg \max _{x_{i} \in \mathcal{L}} p\left(y_{i} \mid x_{i}\right) \mid \forall i \in \mathcal{S}\right\} .
$$

\section{SENSITIVIty ANALYSES OF THE MRF PARAMETERS}

There are four free parameters in the MRF prior model presented in Section II-C, namely, $\sigma_{g}$ in (5), $\sigma_{h}$ in (6), $\beta_{1}$, and $\beta_{2}$ in (3). The parameters $\sigma_{g}$ and $\sigma_{h}$ define the desired structural locality and the amount of orientation discrepancy filtering among

\footnotetext{
${ }^{6}$ Theoretically, the SA algorithm is capable of converging to the global minimum. Geman and Geman [42] proved the existence of annealing schedules which guarantee such convergence. However, these annealing schedules have practical weakness - they are too slow to be used-as pointed out by the authors. In practice, heuristic and faster schedules are employed instead, which may lead SA converges to a local minimum [22].
} 
neighboring voxels, respectively. Plausible values of the parameters $\sigma_{g}$ and $\sigma_{h}$ are suggested in this paragraph. To compromise between the computational speed and the robustness of the algorithm, a $3 \times 3 \times 3$ neighborhood system is used in the ICM algorithm. This leads to a justifiable choice to set $\sigma_{g}=1$. For the orientation discrepancy filtering, we suggest $\sigma_{h}=0.2$. This implies that the algorithm has a 95\% cutoff at a discrepancy measure which equals $2 \sigma_{h}=0.4$. In other words, the algorithm filters out neighboring voxels that are located outside the capture range of the filter, $\pm 53^{\circ}$ deviation from the two principal directions of the iso-surface planar patch at each voxel, i.e., $\hat{\vec{w}}_{1}$ and $\hat{\vec{w}}_{2}$.

In addition, the relationship between the parameters $\beta_{1}$ and $\beta_{2}$ has been studied. To recapitulate, $\beta_{1}$ and $\beta_{2}$ control the influence of orientation coherence in the interactions among neighboring voxels. The orientation is depicted by the first and the second principal directions of the iso-surface planar patch at each voxel. Fig. 8(a) and (b) shows the two approximated principal directions of the noiseless synthetic torus, i.e., $\hat{\vec{w}}_{1}$ and $\hat{\vec{w}}_{2}$, respectively.

In the study, the synthetic torus image (i.e., the torus mentioned in Section III-C) with the additive white Gaussian noise at $\mathrm{SNR}=5$ has been tested with the proposed algorithm. The GU mixture model is employed in the observation model. The Gaussian component aims to model the background intensity values, while the uniform component is used to model the $o b$ ject intensity values, which corresponds to a parabolic intensity profile. The algorithm with parameters configuration: $\sigma_{g}=1$, $\sigma_{h}=0.2$ and the different values of $\beta_{1}$ and $\beta_{2}$ (from $0-10$ with a step size of 0.5 ) have been tested.

Fig. 9(a) presents the study findings. The vertical axis of the graph shows the Jaccard similarity coefficient (JSC) between the estimated and the truth segmentations. JSC is defined as the ratio of the size of the intersection volume to the size of the union volume of the two given segmentations [48]. It is used to quantify the accuracy of an estimated segmentation. JSC gives value 1 if the estimated segmentation equals the truth segmentation. From the figure, it is observed that the parameters $\beta_{1}$ and $\beta_{2}$ complement each other.

In further studies, our algorithm (parameters $\sigma_{g}=1, \sigma_{h}=$ $0.2, \beta_{1}=3$ and $\beta_{2}=2$ ) has been tested on the synthetic images of toruses in different radii (1 voxel, 3, 5, 7, and 10 voxels) corrupted by different levels of additive white Gaussian noise ( $\mathrm{SNR}=1,2, \ldots, 10)$. The experimental results are presented in Fig. 9(b). It is evident that the algorithm is robust to noise (for SNR $\geq 3$ ) over a wide range of object sizes (5-10 voxels in radius). For small objects with radius of 3 voxels, the algorithm can give a satisfactory estimation if SNR $\geq 4$, whereas the segmentation of tiny objects (i.e., 1 voxel in radius) is fair.

Fig. 10(a) shows the binary segmentation of the noisy $(\mathrm{SNR}=5)$ ring torus produced by our algorithm with $\sigma_{g}=1$, $\sigma_{h}=0.2, \beta_{1}=3$, and $\beta_{2}=2$, as compared to the segmentation obtained if either the parameter $\beta_{1}$ or $\beta_{2}$ is vanished [shown in Fig. 10(b) and (c), respectively]. The segmentation presented in Fig. 10(a) contains low-intensity regions of the torus (i.e., regions that are close to the edge of the torus), which are partially left out in the other two segmentations.

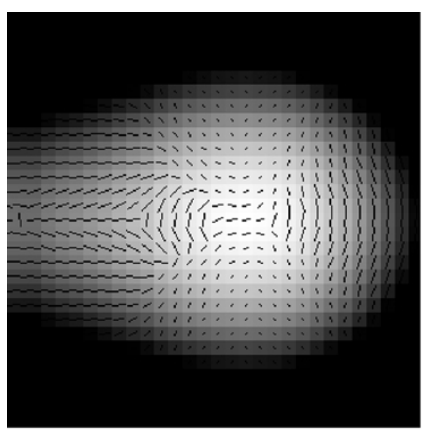

(a) $\hat{\overrightarrow{w_{1}}}$

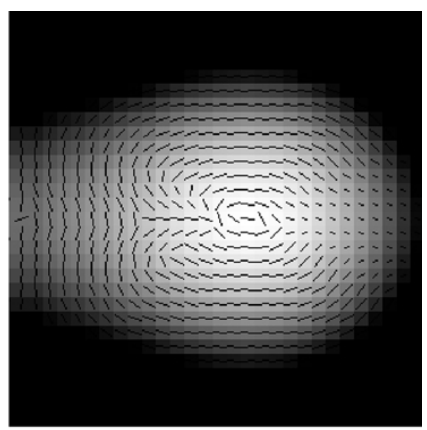

(b) $\hat{\vec{w}_{2}}$
Fig. 8. Local structural orientation estimated. (a) The first principal directions of the iso-surface planar patches, AKA the directions of minimum curvature $\hat{\vec{w}}_{1}$ and (b) the second principal directions of the iso-surface planar patches, AKA the directions of maximum curvature $\hat{\vec{w}}_{2}$

This suggests that ignoring the orientation coherence in either direction will adversely affect the quality of segmentation. For this particular synthetic image, setting $\beta_{1}$ to zero has a greater degeneration of the binary segmentation. Because of the complementary behavior of the two free parameters $\beta_{1}$ and $\beta_{2}$, we suggest that they should be assigned to comparable nonzero values, in order to yield satisfactory binary segmentation.

\section{EXPERIMENTS AND DISCUSSION ON THE PERFORMANCE OF THE ALGORITHM}

In this section, the experimental results on the synthetic and the real-world medical images are presented. A discussion on the performance of the algorithm follows.

\section{A. Experiments on Synthetic Images}

We have compared the proposed algorithm (parameter s configuration: $\sigma_{g}=1, \sigma_{h}=0.2, \beta_{1}=3$, and $\beta_{2}=2$ ) with the global thresholding based on ML estimation (i.e., without any smoothness prior) and the algorithm with the multilevel logistic (MLL) MRF model (i.e., with only simple all-directional smoothness constraint). The noisy ring torus with $\mathrm{SNR}=5$ is used in the study. Fig. 11 shows the segmentations obtained from our method and the other two algorithms. Fig. 11(a) shows an image slice of the segmented torus produced by our novel algorithm. The JSC value equals 0.87 with respect to the ground truth. Fig. 11(b) and (c) shows the segmentations of the ring torus obtained from the global thresholding and the algorithm with the MLL MRF model, respectively. Compared with our algorithm, their JSC values are relatively low, which are both equal to 0.39 only. It is observed that the segmentation produced by the global thresholding contains quite a few holes, especially in the low-intensity regions of the ring torus, whereas the algorithm with the MLL MRF model is unable to segment the low-intensity regions from the background.

\section{B. Experiments on Real-World Medical Images}

In addition to the experiments on the synthetic images, the novel algorithm has been tested on real-world images. It has been applied to two medical images obtained from the Department of Diagnostic Radiology and Organ Imaging, Prince of 


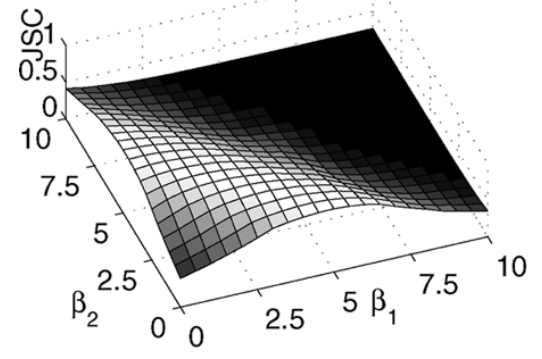

(a) $\beta_{1}$ and $\beta_{2}$ varying

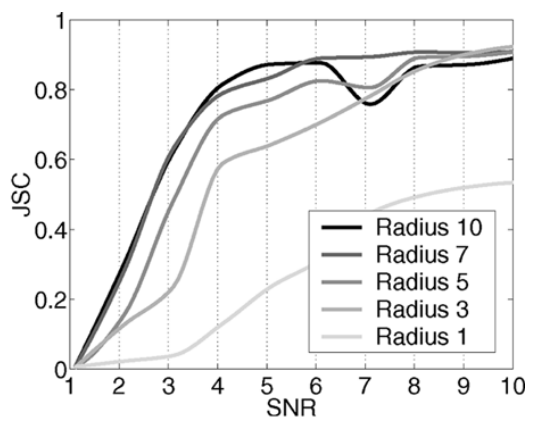

(b) $\beta_{1}=3$ and $\beta_{2}=2$

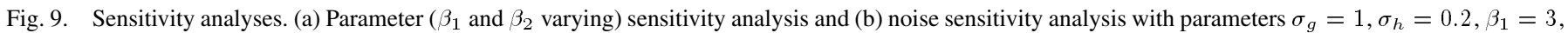
and $\beta_{2}=2$ for the ring toruses in different tube radii 1 voxel (lowest curve at SNR $=5$ ), 3, 5, 7, and 10 voxels.

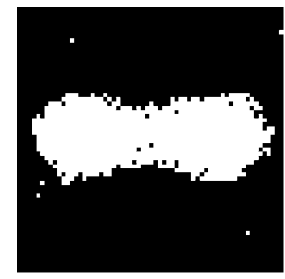

(a) $\beta_{1}=3, \beta_{2}=2$

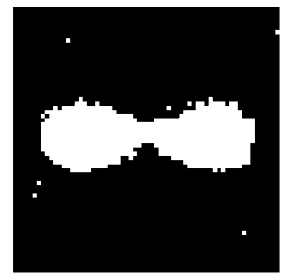

(b) $\beta_{1}=0, \beta_{2}=2$

Fig. 10. Noisy $(\mathrm{SNR}=5)$ ring torus segmentation. Segmentation produced by our novel algorithm with parameters (a) $\beta_{1}=3, \beta_{2}=2$, (b) $\beta_{1}=0, \beta_{2}=2$ and (c) $\beta_{1}=3$, and $\beta_{2}=0$.

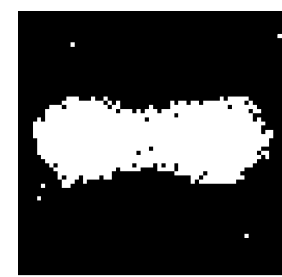

(a) With orientation

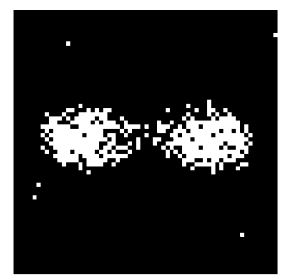

(b) Global thresholding

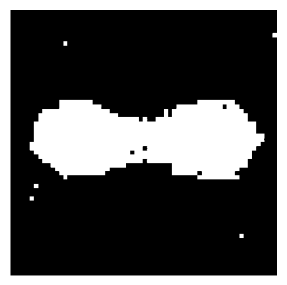

(c) $\beta_{1}=3, \beta_{2}=0$

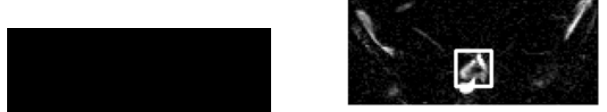

(a) $16^{t h}$ slice image

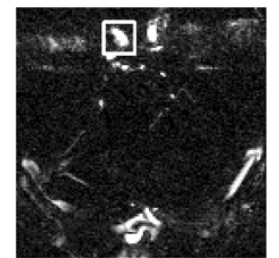

(a) $15^{\text {th }}$ slice image
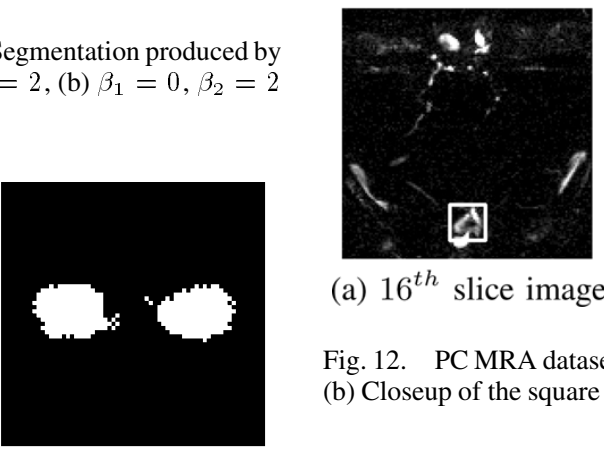

(c) MLL MRF Model

Fig. 11. Noisy ( $\mathrm{SNR}=5$ ) ring torus segmentation. Segmentation produced by (a) our novel algorithm, (b) the global thresholding with the ML estimation, and (c) the algorithm with the MLL MRF model.

Wales Hospital, Hong Kong. The two images are PC MRA intracranial scans that were acquired from a Siemens ${ }^{7} 1.5 \mathrm{~T}$ Sonata imager. The data volume is $256 \times 176 \times 30$ voxels with a voxel size of $0.9 \times 0.9 \times 1.5 \mathrm{~mm}^{3}$.

PC MRA is one of the noninvasive imaging modalities that can provide accurate 3 -D vascular information of a patient. It is one of the most widely available vascular imaging techniques in the clinical environment. The speed image of PC MRA provides information of the patients' blood flow. The intensity values in the image are proportional to the flow velocity. Because of the blood viscosity, frictional force slows down the blood flow near the vascular wall [24]. As such, the intensity profile is nonuniform within the vascular structures. The intensity value is relatively low at the boundary of vessels in the angiogram, while the intensity value is high near the center of the vessels. The intensity inhomogeneity is a challenge if the vascular segmentation is to be robust.

\footnotetext{
${ }^{7}$ Siemens Medical Solutions, Siemens AG.
}

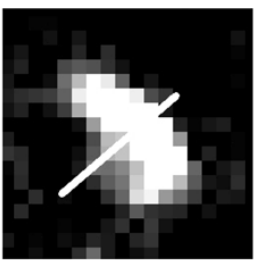

(b) Close-up

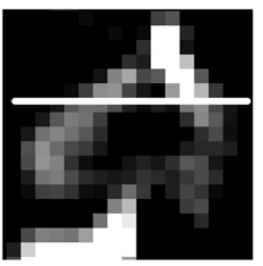

(b) Close-up

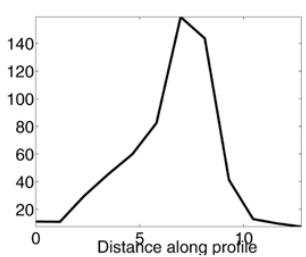

(c) Profile

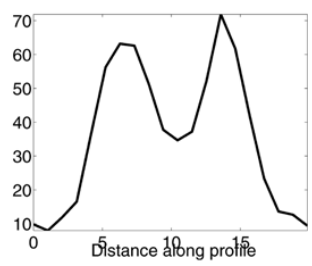

(c) Profile
Fig. 12. PC MRA dataset 1 . The 15 th and 16 th slice images. (a) A slice image. (b) Closeup of the square region in (a). (c) Intensity profile along the line in (b).

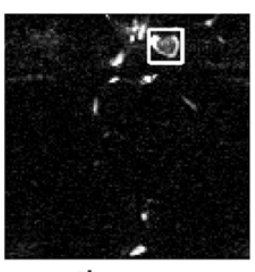

(a) $15^{\text {th }}$ slice image

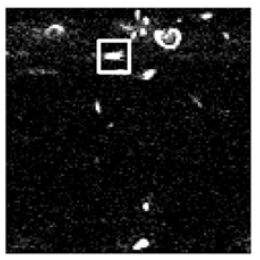

(a) $16^{\text {th }}$ slice image

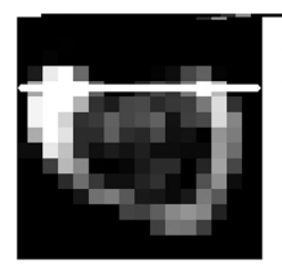

(b) Close-up

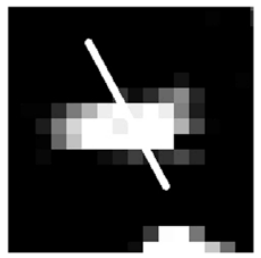

(b) Close-up

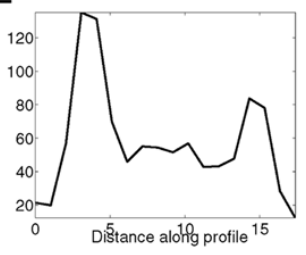

(c) Profile

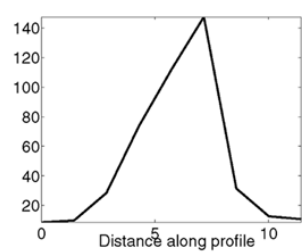

(c) Profile
Fig. 13. PC MRA dataset 2. The 15 th and 16 th slice images. (a) A slice image (b) Closeup of the square region in (a). (c) Intensity profile along the line in (b).

Figs. 12 and 13 show the 15 th and 16th slice images of the two PC MRA datasets, respectively. For each figure, (a) shows a slice image and (b) shows the closeup of the square region in subfigure (a). The intensity profile along the straight line in (b) is shown in (c). It is observed that there is intensity inhomogeneity within the vascular structures in the angiograms, especially in 
the low blood regions near the vascular boundary and inside the aneurysms.

Segmentation of the PC MRA speed images can facilitate an effective and efficient diagnostic review of the vascular information in an angiogram, which helps the physician to define the character and extent of a vascular disease, thereby aiding diagnosis and prognosis. Moreover, segmentation is the first step for other post-processing routines or analyses, such as visualization, volumetric measurement, quantitative comparison, and image-guided surgery [49]. Therefore, we are interested in the application of our segmentation algorithm to the PC MRA speed images.

Experimental results on the two PC MRA datasets are shown in Figs. 14 and 15, respectively (with the regions of interest are defined). For each figure, (a) shows a volume rendered image of the corresponding dataset with the aneurysm highlighted by the arrow, and (b)-(e) show the segmentations obtained from the global thresholding with the ML estimation, the algorithm with the MLL MRF model, the proposed algorithm and a manual delineation by a consultant interventionist who has 15 years of experience in endovascular treatments. By treating the manual delineations of the vessels as the truth segmentations, JSC values of the aforementioned former three segmentations are calculated. On average, the JSC value equals 0.84 for the proposed algorithm, 0.51 for the global thresholding, and 0.43 for the algorithm with the MLL MRF model. It is noticed that the global thresholding and the algorithm with the MLL MRF model cannot segment some of the major vessels and the aneurysms, which are the radiologists' objects of interest.

In the experiments, the parameter configurations were: $\sigma_{g}=$ $1, \sigma_{h}=0.2$ and $\beta_{1}=\beta_{2}=2.5$. The MGU mixture model was used as the observation model, as suggested in [50]. On average, the algorithm takes $42 \mathrm{~s}$, needs $<20$ iterations to converge, and consumes $<100 \mathrm{MB}$ of memory to segment the two PC MRA datasets on a $2.66 \mathrm{GHz} \mathrm{PC}$.

\section{Discussion on the Performance of the Algorithm}

In the experiments on both the synthetic and real-world medical images, we found that the segmentations produced by the newly proposed algorithm are in a high degree of agreement with the truth segmentations. On the contrary, the global thresholding with the ML estimation and the algorithm with the MLL MRF model give less than satisfactory results. They have difficulties in segmenting the low-intensity regions in the images.

One may observe that there is a few single voxel misclassifications on the background in Fig. 11(a), which can also be found in the segmentation obtained with the global thresholding. The reason for the misclassifications is that the structural smoothness constraint is not extended to the background class, as discussed in Section II-C. Owing to the fact that the background is structureless, only piecewise continuous object label assignment is encouraged in the formulation of the prior model [see (3)].

In the approximation of the voxel label assignments, the sum of the likelihood and the prior energy functions have to be minimized. As shown in Section II-C, the prior energy term in (1) vanishes if all the neighbors' labels are assigned to background.

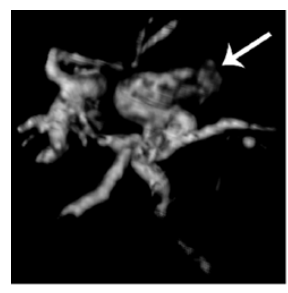

(a) VR image

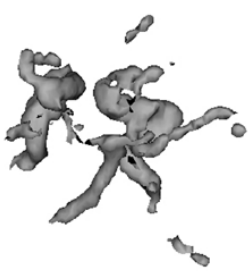

(b) Global thresholding

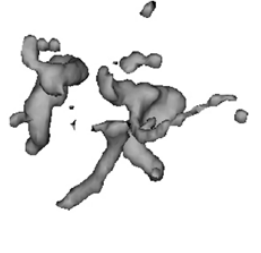

(c) MLL MRF model

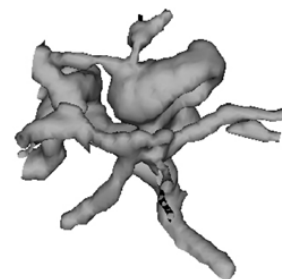

(d) With orientation

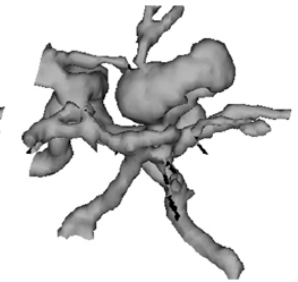

(e) Manual
Fig. 14. PC MRA dataset 1. (a) Volume rendered image, the aneurysm is highlighted by an arrow; segmentations obtained from (b) the global thresholding with the ML estimation, (c) the algorithm with the MLL MRF model, (d) the proposed algorithm, and (e) a manual delineation by an experienced consultant radiologist.

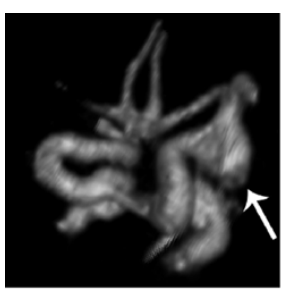

(a) VR image

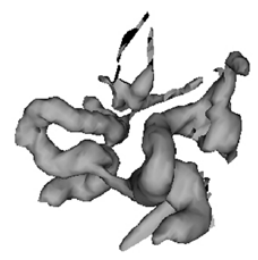

(b) Global thresholding

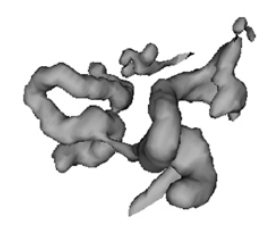

(c) MLL MRF model

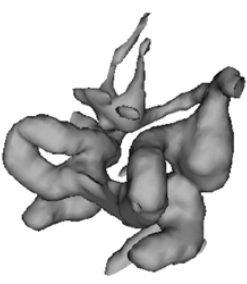

(d) With orientation

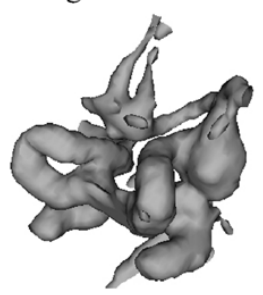

(e) Manual
Fig. 15. PC MRA dataset 2. (a) Volume rendered image the aneurysm is highlighted by an arrow; segmentations obtained from (b) the global thresholding with the ML estimation, (c) the algorithm with the MLL MRF model, (d) the proposed algorithm, and (e) a manual delineation by an experienced consultant radiologist.

As a result, the label assigned to the voxel becomes solely dependent on the likelihood energy function. As such, in the case of a high-intensity noise that stands out on the background, a single voxel misclassification is expected. However, this type of misclassification can be removed easily with a connectivity filter. Therefore, we believe that the single voxel misclassification on the background is not a problem. On the contrary, it is a big problem if the low-intensity region can not be segmented in an angiogram. As compared with the other two algorithms, the experiments on real-world images have demonstrated that our novel method is robust to the low-intensity region and is capable of segmenting the region in the angiograms. 
A few (2-3 voxels in diameter) dim vascular structures are left out in the segmentations obtained with our algorithm [see Figs. 14(d) and 15(d)] as compared with the manual segmentations [see Figs. 14(e) and 15(e)]. However, these dim vascular structures correspond to the nonmajor vessels, which is not the radiologists' primary interest at the current work. According to the radiologists' feedback, these results are good enough for clinical applications. The objects of interest (i.e., aneurysms) are well segmented in the segmentations produced by our algorithm.

We have demonstrated that dim vascular structure with coherent intensity profile (e.g., an aneurysm or the boundary of vessel) can be segmented in an angiogram with the application of the proposed structural smoothness constraint. It is worth mentioning that the dim vascular structure usually occupies only a few voxels in the angiogram, which poses difficulties if one wants to model the coherence in image data surface explicitly. As has been pointed out in [25] and [27], the explicit modeling technique requires a large region (more than 10-30 pixels) for the modeling to be robust. This makes our approach more preferable to the explicit modeling approach as proposed in [25]-[27].

\section{CONCLUSION}

We have presented a novel image segmentation technique to segment the objects with coherent image data surface. The method that we have proposed uses a new smoothness prior, which exploits the coherence of the local structural orientation in the image to constrain the segmentation process. The prior is expressed as a function of geometric closeness and structural orientation similarity measures. We have also described a method to estimate the local structural orientation with an orientation tensor. This method is demonstrated to be more robust than the conventional Hessian matrix. The experiments on the synthetic images have shown that the orientation tensor approach outperforms its conventional counterpart in terms of estimation accuracy and robustness to noise.

Our algorithm has been applied to synthetic images and realworld medical images. The experimental results have indicated that the new method produces better segmentations than the global thresholding with the ML estimation and the algorithm with the MLL MRF model. Moreover, the segmentations in the real-world medical images obtained by our method are comparable to the manual segmentations from an experienced consultant radiologist.

In this work, we have introduced an application of the local structural orientation smoothness prior to the segmentation of 3-D medical images. We expect the application of this prior to be extended naturally to other areas such as image restoration with edge-preserving or coherent-enhancing capability (see [51], [52], and references therein), scientific image segmentation [39], and object extraction from video [53].

Several possible improvements to the proposed method are of interest for future research. An extension to the scale-space is worth studying because a multiscale analysis of the local structural orientation may allow the algorithm to produce robust segmentations of objects in a variety of scales. Furthermore, a coarse to fine strategy for image segmentation is possible if the
Bayesian segmentation is performed in a multigrid fashion. This may further increase the algorithm's robustness to noise and improve the efficiency of the proposed method.

\section{ACKNOWLEDGMENT}

The authors would like to thank Prof. C.-F. Westin for the fruitful discussions and for providing MATLAB codes on the construction of the quadrature filters at the early stage of the software development. They would also like to thank Dr. Yu of the Department of Diagnostic Radiology and Organ Imaging, Prince of Wales Hospital, Hong Kong, for providing the clinical data and helping the segmentation, as well as the anonymous reviewers for their perceptive comments, which have significantly improved this paper.

\section{REFERENCES}

[1] R. C. Gonzalez and R. E. Woods, Digital Image Processing, 2nd ed. Reading, MA: Addison-Wesley, 2002.

[2] N. R. Pal and S. K. Pal, "A review on image segmentation techniques," Pattern Recognit., vol. 26, no. 9, pp. 1277-1294, 1993.

[3] J. S. Suri, K. Liu, S. Singh, S. N. Laxminarayan, X. Zeng, and L. Reden, "Shape recovery algorithms using level sets in 2-D/3-D medical imagery: A state-of-the-art review," IEEE Trans. Inf. Technol. Biomed., vol. 6, no. 1, pp. 8-28, Mar. 2002.

[4] H. Trichili, M. S. Bouhlel, and F. Kammoun, "A review and evaluation of medical image segmentation using methods of optimal filtering," $J$. Test. Eval., vol. 31, no. 5, pp. 398-404, 2003.

[5] T. McInerney and D. Terzopoulos, "Topology adaptive deformable surfaces for medical image volume segmentation," IEEE Trans. Med. Imag., vol. 18, no. 6, pp. 840-850, Jun. 1999.

[6] L. M. Lorigo, O. Faugeras, W. E. L. Grimson, R. Keriven, R. Kikinis, and C.-F. Westin, "Co-dimension 2 geodesic active contours for MRA segmentation," in Proc. Int. Conf. Information Processing in Medcal Imaging, 1999, pp. 126-139.

[7] K. C. Wang, R. W. Dutton, and C. A. Taylor, "Improving geometric model construction for blood flow modeling," IEEE Eng. Med. Biol. Mag., vol. 18, no. 6, pp. 33-39, Jun. 1999.

[8] C.-F. Westin, L. M. Lorigo, O. Faugeras, W. E. L. Grimson, S. Dawson, A. Norbash, and R. Kinkinis, "Segmentation by adaptive geodesic active contours," in Medical Image Computing and Computer-Assisted Intervention. Berlin, Germany: Springer-Verlag, 2000, vol. 1935, pp. $266-275$.

[9] H. T. Nguyen, M. Worring, and R. van den Boomgaard, "Watersnakes: Energy-driven watershed segmentation," IEEE Trans. Pattern Anal. Mach. Intell., vol. 25, no. 3, pp. 330-342, Mar. 2003.

[10] J. McLachlan and D. Peel, Finite Mixture Models. New York: Wiley, 2000.

[11] J. K. Fwu and P. M. Djurić, "Unsupervised vector image segmentation by a tree structure-ICM algorithm," IEEE Trans. Med. Imag., vol. 15, no. 6, pp. 871-880, Jun. 1996.

[12] Y. W. Lim and S. U. Lee, "On the color image segmentation algorithm based on the thresholding and the fuzzy c-means technique," Pattern Recognit., vol. 23, no. 9, pp. 935-952, 1990.

[13] M. R. Rezaee, P. M. J. van der Zwet, B. P. F. Lelieveldt, R. J. van der Geest, and J. H. C. Reiber, "A multiresolution image segmentation technique based on pyramidal segmentation and fuzzy clustering," IEEE Trans. Image Process., vol. 9, no. 10, pp. 1238-1248, Oct. 2000.

[14] M. N. Ahmed, S. M. Yamany, N. Mohamed, A. A. Farag, and T. Moriarty, "A modified fuzzy c-means algorithm for bias field estimation and segmentation of MRI data," IEEE Trans. Med. Imag., vol. 21, no. 2, pp. 193-199, Feb. 2002.

[15] T. F. Chan and L. A. Vese, "Active contours without edges," IEEE Trans. Image Process., vol. 10, no. 2, pp. 266-277, Feb. 2001.

[16] L. Liu and S. Sclaroff, "Region segmentation via deformable modelguided split and merge," in Proc. Int. Conf. Computer Vision, vol. 1, 2001, pp. 98-104.

[17] Y. Wang, T. Adali, J. Xuan, and Z. Szabo, "Magnetic resonance image analysis by information theoretic criteria and stochastic site models," IEEE Trans. Inf. Technol. Biomed., vol. 5, no. 1, pp. 150-158, Mar. 2001. 
[18] M. Egmont-Petersen, D. de Ridder, and H. Handels, "Image processing with neural networks-A review," Pattern Recognit., vol. 35, pp. 2279-2301, 2002

[19] S. Ruan, B. Moretti, J. Fadili, and D. Bloyet, "Fuzzy Markovian segmentation in application of magnetic resonance images," Comput. Vis. Image Understand., vol. 85, pp. 54-69, 2002.

[20] S. Y. Wan and W. E. Higgins, "Symmetric region growing," IEEE Trans. Image Process., vol. 12, no. 9, pp. 1007-1015, Sep. 2003.

[21] C. D'Elia, G. Poggi, and G. Scarpa, "A tree-structured Markov random field model for Bayesian image segmentation," IEEE Trans. Image Process., vol. 12, no. 9, pp. 1259-1273, Oct. 2003.

[22] S. Z. Li, Markov Random Field Modeling in Image Analysis, 2nd ed. Tokyo, Japan: Springer-Verlag, 2001.

[23] H. Guillemaud and M. Brady, "Estimating the bias field of MR images," IEEE Trans. Med. Imag., vol. 16, no. 2, pp. 238-251, Feb. 1997.

[24] Y. C. Fung, Biomechanics: Circulation, 2nd ed. New York: SpringerVerlag, 1996.

[25] P. J. Besl and R. C. Jain, "Segmentation through variable-order surface fitting," IEEE Trans. Pattern Anal. Mach. Intell., vol. 10, no. 2, pp. 167-192, Feb. 1988

[26] Y. G. Leclerc, "Constructing simple stable description for image partitioning," Int. J. Comput. Vis., vol. 3, no. 1, pp. 73-102, 1989.

[27] Z. Tu and S. C. Zhu, "Image segmentation by data-driven Markov chain Monte Carlo," IEEE Trans. Pattern Anal. Mach. Intell., vol. 24, no. 5, pp. 657-673, May 2002.

[28] D. Geiger and F. Girosi, "Parallel and deterministic algorithms from MRF's: Surface reconstruction," IEEE Trans. Pattern Anal. Mach. Intell., vol. 13, no. 5, pp. 401-412, May 1991.

[29] J. Besag, "On the statistical analysis of dirty pictures," J. Roy. Stat. Soc. $B$, vol. 48, no. 3, pp. 259-302, 1986.

[30] C. Tomasi and R. Manduchi, "Bilateral filtering for gray and color images," in Proc. Int. Conf. Computer Vision, 1998, pp. 839-846.

[31] H. Knutsson, "Representing local structure using tensors," in Proc. Scandinavian Conf. Image Analysis, 1989, pp. 244-251.

[32] G. H. Granlund and H. Knutssan, Signal Processing for Computer Vision. Norwell, MA: Kluwer, 1995.

[33] Y. Sato, S. Nakajima, N. Shiraga, H. Atsumi, S. Yoshida, T. Koller, G. Gerig, and R. Kikinis, "3-D multi-scale line filter for segmentation and visualization of curvilinear structures in medical images," Med. Imag. Anal., vol. 2, no. 2, pp. 143-168, 1998.

[34] S. Aylward and E. Bullitt, "Initialization, noise, singularities, and scale in height ridge traversal for tubular object centerline extraction," IEEE Trans. Med. Imag., vol. 21, no. 1, pp. 61-75, Jan. 2002.

[35] K. Krissian, G. Maladain, R. Vaillant, Y. Trousset, and N. Ayache, "Model-based multiscale detection of 3-D vessels," Comput. Vis. Pattern Recognit., pp. 722-727, 1998.

[36] S. Lakshmanan and H. Derin, "Simultaneous parameter estimation and segmentation of Gibbs random fields using simulated annealing," IEEE Trans. Pattern Anal. Mach. Intell., vol. 11, no. 8, pp. 799-813, Aug. 1989.

[37] Z. Kato, J. Zerubia, and M. Berthod, "Unsupervised parallel image classification using Markovian models," Pattern Recognit., vol. 32, pp. 591-604, 1999.

[38] J. M. Laferté, P. Pérez, and F. Heitz, "Discrete Markov image modeling and inference on the quatree," IEEE Trans. Image Process., vol. 9, no. 4, pp. 390-404, Apr. 2000.

[39] M. Mignotte, C. Collet, P. Perez, and P. Bouthemy, "Sonar image segmentation using an unsupervised hierarchical MRF model," IEEE Trans. Image Process., vol. 9, no. 10, pp. 1216-1231, Oct. 2000.

[40] R. Wilson and C. T. Li, "A class of discrete multiresolution random fields and its application to image segmentation," IEEE Trans. Pattern Anal. Mach. Intell., vol. 25, no. 1, pp. 42-56, Jan. 2003

[41] Y. Boykov, O. Veksler, and R. Zabih, "Fast approximate energy minimization via graph cuts," IEEE Trans. Pattern Anal. Mach. Intell., vol. 23 , no. 11 , pp. $1222-1239$, Nov. 2001.

[42] S. Geman and D. Geman, "Stochastic relaxation, Gibbs distributions, and the Bayesian restoration of images," IEEE Trans. Pattern Anal. Mach. Intell., vol. PAMI-6, no. 6, pp. 721-741, Jun. 1984.
[43] A. Blake and A. Zisserman, Visual Reconstruction. Cambridge, MA: MIT Press, 1987.

[44] C. Peterson and B. Soderberg, "A new method for mapping optimization problems onto neural networks," Int. J. Neur. Syst., vol. 1, no. 1, pp. 3-22, 1989.

[45] D. J. Miller and Q. Zhao, "A sequence-based extension of mean-field annealing using the forward/backward algorithm: Application to image segmentation," IEEE Trans. Signal Process., vol. 51, no. 12, pp. 2692-2705, Dec. 2003.

[46] J. L. Marroquin, E. A. Santana, and S. Botello, "Hidden Markov measure field models for image segmentation," IEEE Trans. Pattern Anal. Mach. Intell., vol. 25, no. 12, pp. 1380-1387, Dec. 2003.

[47] H. Ishikawa, "Exact optimization for Markov random fields with convex priors," IEEE Trans. Pattern Anal. Mach. Intell., vol. 25, no. 10, pp. 1333-1336, Oct. 2003.

[48] K. V. Leemput, F. Maes, D. Vandermeulen, A. Colchester, and P. Suetens, "Automated segmentation of MS lesions from multi-channel MR images," in Medical Image Computing and Computer Assisted Intervention. Berlin, Germany: Springer-Verlag, 1999, vol. 1679, pp. $11-21$.

[49] J. S. Suri, K. Liu, L. Reden, and S. Laxminarayan, "A review on MR vascular image processing algorithms: Acquisition and prefiltering: Part I," IEEE Trans. Inform. Technol. Biomed., vol. 6, no. 2, pp. 324-337, Jun. 2002.

[50] A. C. S. Chung, J. A. Noble, and P. Summers, "Fusing speed and phase information for vascular segmentation of phase contrast MR angiograms," Med. Imag. Anal., vol. 6, no. 2, pp. 109-128, 2002.

[51] J. Weickert, "Coherence-enhancing diffusion filtering," Int. J. Comput Vis., vol. 31, no. 2-3, pp. 111-127, 1999.

[52] D. Barash, "A fundamental relationship between bilateral filtering, adaptive smoothing, and the nonlinear diffusion equation," IEEE Trans. Pattern Anal. Mach. Intell., vol. 24, no. 6, pp. 844-847, Jun. 2002.

[53] Y. T. Cui and Q. Huang, "Character extraction of license plates from video," Comput. Vis. Pattern Recognit., pp. 502-507, 1997.

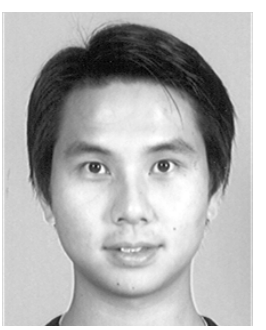

Wilbur C. K. Wong received the B.Eng. degree (first class honors) and the M.Phil. degree in computer science from the Hong Kong University of Science and Technology. He is currently pursuing the Ph.D. degree in computer science under the supervision of Dr. A. C. S. Chung.

His research interests include image processing and medical image analysis.

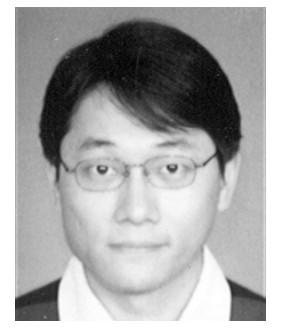

Albert C. S. Chung received the B.Eng. degree (firstclass Honors) in computer engineering from the University of Hong Kong in 1995 and the M.Phil. degree in computer science from the Hong Kong University of Science and Technology in 1998.

He joined the Medical Vision Laboratory, University of Oxford, Oxford, U.K., as a doctoral research student with the Croucher Foundation scholarship and graduated in 2001. He was a Visiting Scientist at the Artificial Intelligence Laboratory, Massachusetts Institute of Technology, Cambridge, in 2001. He is currently and Assistant Professor with the Department of Computer Science, Hong Kong University of Science and Technology. His research interests include medical image analysis, image processing, and computer vision.

Dr. Chung won the 2002 British Machine Vision Association Sullivan Thesis Award for the best doctoral thesis submitted to a U.K. university in the field of computer or natural vision. 